\title{
Técnicas de aplicação de fungicida em trigo para o controle de giberela (Gibberella zeae) ${ }^{1}$
}

\author{
Fungicidal spray techniques for the control of head blight \\ (Gibberella zeae) in wheat
}

\section{Edivan Panisson ${ }^{2}$ Walter Boller ${ }^{3}$ Erlei Melo Reis ${ }^{4}$ Laércio Hoffmann $^{5}$}

\section{RESUMO}

Durante muitos anos, a giberela, causada pelo fungo Gibberella zeae (anamorfo Fusarium graminearum), foi considerada de importância secundária no sul do Brasil. O aumento da intensidade e da freqüencia de ocorrência, tornou a giberela uma das doenças de maior importância na cultura do trigo. A giberela é uma doença de infecção floral e mesmo os fungicidas sistêmicos recomendados apresentam apenas efeito protetor das anteras. Os objetivos do presente trabalho foram avaliar a eficiência de controle e os efeitos nos grãos colhidos, de dois tipos de pontas de pulverização (leque e duplo leque), diferentes arranjos dos bicos na barra de aplicação e dois volumes de calda. A desuniformidade da antese aparece como um dos principais fatores envolvidos com a baixa eficiência dos fungicidas. Nos experimentos, realizados no ano 2000, a aplicação dos tratamentos reduziu significativamente a incidencia, o número de espiguetas gibereladas e a severidade da doença, aumentando o rendimento de grãos. As pontas de pulverização, seus arranjos na barra e os volumes de calda utilizados comportaram-se de maneira semelhante em todas as variáveis avaliadas. $O$ incremento no rendimento de grãos, obtido em relação à testemunha, sugere que se deve recomendar a aplicação de fungicidas para o controle da giberela, utilizando pontas que geram gotas finas a médias com volume de calda de 200 L.ha'
Palavras-chave: deposição de gotas, barra modificada, pontas de pulverização.

\section{ABSTRACT}

For many years, the head blight of winter cereal crops, caused by the fungus Gibberella zeae, was considered a disease of secondary importance. However, the increased frequency and yield losses associated with head blight in Southern Brazil have turned this disease into a major problem. Gibberella zeae enters the host plants through the anthers. To avoid infection, the fungicides need to protect the anthers from the fungus, which requires improved spray techniques. The role of two types of spray nozzles (single jet and twin jet), different nozzle arrangements, and two spray volumes on head blight control and grain yield were evaluated. The experiments conducted at the field, during the year 2000, showed that the fungicidal applications reduced disease incidence, the number of infected spikelets, and disease severity significantly. The grain yield was increased as well. The spray nozzles, nozzle arrangements, and spray volumes did not influence disease control and grain yield. The differences in grain yield between treated and non-treated plots made chemical control worthy, despite its low efficacy. Fungicidal sprays to control head blight should use nozzles that deliver medium to fine droplets, at a volume of 200 L.ha'.

\footnotetext{
${ }^{1}$ Parte da dissertação de mestrado do primeiro autor, apresentada ao Programa de Pós- graduação em Agronomia da Faculdade de Agronomia e Medicina Veterinária da Universidade de Passo Fundo (FAMV/UPF).

${ }^{2}$ Engenheiro Agrônomo Mestre em Agronomia A/C Fitopatologia

${ }^{3}$ Engenheiro Agrônomo, Dr., Docente da FAMV/UPF, orientador da dissertação. C.P.611, 99001-970, Passo Fundo, RS. Telefone/ Fax: (0XX) 316-8151. E-mail: boller@upf.tche.br.Autor para correspondência.

${ }^{4}$ Engenheiro Agrônomo, PhD, docente da FAMV/UPF, co-orientador da dissertação.

${ }^{5}$ Engenheiro Agrônomo aluno do Programa de pós-graduação em Agronomia da FAMV/UPF.
} 
Key words: droplet deposition, spray nozzles, modified spray boom.

\section{INTRODUÇÃO}

O principal agente causal da giberela nos cereais de inverno é o fungo Gibberella zeae (Schw.) Petch., anamorfo Fusarium graminearum (Schwabe). Nos últimos anos, tem sido observado aumento da intensidade e da freqüência da doença em trigo (Triticum aestivum L.) em muitas regiões onde este cereal é cultivado (Mc MULLEN et al., 1997). A giberela é uma doença de infecção floral e as epidemias ocorrem associadas com períodos prolongados de chuva ou alta umidade durante a antese do trigo (SUTTON, 1982; 1988; PARRY et al., 1995). WIESE, 1987 relatou que temperatura do ar superior a $18{ }^{\circ} \mathrm{C}$ e duração do molhamento das espigas acima de $72 \mathrm{~h}$ são condições que estimulam o desenvolvimento da doença.

Os danos causados pela doença são quantitativos e qualitativos (WIESE, 1987; REIS, 1988; BAI \& SHANER, 1994).

AGRIOS (1988) e SUTY \& MAULER-

MACHNIK (1996) relataram que sob condições epidêmicas podem ser observadas reduções no rendimento de trigo de até $50 \%$. Na China, em anos com epidemias severas (50 a 100\% de incidência), foram detectadas reduções no rendimento de 10 a $40 \%$ (BAI \& SHANER, 1994). No Brasil, a doença ocorre principalmente nos estados da região sul e os maiores danos registrados foram de $14 \%$, porém a média em 10 anos de experimentos foi de 5,4\% (REIS et al., 1996). Os principais danos qualitativos causados pela doença são o acúmulo de micotoxinas nos grãos, a redução do peso do hectolitro, redução do peso de mil grãos e redução do poder germinativo das sementes (PARRY et al., 1995).

Conforme REIS (1988) e Reunião... (2000), o controle químico da giberela deve ser recomendado em lavouras onde as condições climáticas são favoráveis ao estabelecimento do patógeno, levandose em conta a ocorrência da doença nos últimos 10 anos.

As principais dificuldades relacionadas com o controle químico da giberela são: a) esporadicidade de sua ocorrência (REIS, 1988), b) dificuldade de aplicar os fungicidas no momento correto (REIS, 1988; MESTERHÁZY \& BARTÓK, 1996) e c) dificuldade de atingir os sítios de infecção (as anteras) com os fungicidas (REIS et al., 1996).

MAULER-MACHNIK \& ZAHN (1994) e REIS, et al. (1996) afirmaram que para o controle de giberela são necessários fungicidas que apresentem alta eficiência e que o controle seja realizado no momento adequado, quando a quantidade máxima de anteras estão expostas. Isto ocorre, em geral, do $5^{\circ}$ ao $8^{\circ}$ dia após o início da antese, podendo variar de acordo com a cultivar, época de semeadura ou condições ambientais (REIS, 1988; VARGAS et al., 2000).

MAULER-MACHNIK \& ZAHN (1994) salientam que é necessário obter uma boa cobertura das espigas para obter sucesso no controle. Diante disto, é necessário utilizar equipamentos apropriados que forneçam densidade e tamanho de gota adequados para alcançar este objetivo.

O fungicida tebuconazole é um dos ingredientes ativos que apresenta melhor eficiência de controle (MAULER-MACHNIK \& ZAHN, 1994; REIS et al., 1996; SUTY \& MAULER-MACHNIK,1996). Em laboratório, os fungicidas apresentam eficiência superior a $90 \%$, porém no campo ela é inferior a $75 \%$ (Reunião..., 2000), sendo esta redução atribuída à insuficiente deposição dos fungicidas nas anteras.

Para o estudo em questão, o alvo biológico são as anteras (REIS et al., 1996), necessitando-se atingi-las para que o fungicida proporcione controle.

Os objetivos deste trabalho foram avaliar tipos de pontas de pulverização, arranjos dos bicos na barra e volumes de calda em relação ao controle da doença no campo, bem como as características que afetam o rendimento e/ou a qualidade dos grãos

\section{MATERIALEMÉTODOS}

Conduziram-se dois experimentos, na Faculdade de Agronomia e Medicina Veterinária da Universidade de Passo Fundo, na safra de 2000, em duas épocas de semeadura. O trigo, cultivar BR-23, foi semeado nos dias 26 de junho e 08 de julho, respectivamente, com densidade de semeadura de 370 sementes viáveis. $\mathrm{m}^{-2}$. O tamanho das parcelas foi de 2,55 m x $6 \mathrm{~m}$. Os tratos culturais, o manejo de plantas daninhas e de pragas, foram realizados de acordo com as recomendações técnicas para a cultura do trigo (Reunião..., 2000). Para o controle de oídio foi realizada uma aplicação de fungicida triadimenol (125 g.i.a.ha ${ }^{-1}$ ), exceto na testemunha 1 , no dia 07 de setembro. Para garantir condições favoráveis à infecção, foi realizada irrigação, mantendo as espigas molhadas durante a antese.

Os tratamentos utilizados encontram-se descritos na tabela 1, com ilustrações na figura 1. A aplicação dos tratamentos foi realizada no estádio 65 da escala fenológica de ZADOCKS et al. (1974). O fungicida utilizado foi o tebuconazole $200 \mathrm{CE}$, na dose de 150 g.i.a.ha ${ }^{-1}$. A aplicação do fungicida foi realizada 
Tabela 1 - Detalhamento da composição dos tratamentos utilizados para o controle de giberela.

\begin{tabular}{|c|c|c|c|c|c|c|}
\hline Tratamento & Barra & $\begin{array}{l}\text { Forma do } \\
\text { Jato }\end{array}$ & $\begin{array}{c}\text { Ponta de } \\
\text { Pulverização }\end{array}$ & $\begin{array}{l}\text { Pressão } \\
(\mathrm{kPa})\end{array}$ & $\begin{array}{l}\text { Volume } \\
\left(\text { L.ha }^{-1}\right)\end{array}$ & $\begin{array}{c}\mathrm{DMV}^{3} \\
(\mu \mathrm{m})\end{array}$ \\
\hline 1 & Testemunha $1^{*}$ & & & & & \\
\hline 2 & Testemunha $2^{* *}$ & & & & & \\
\hline 3 & Tradicional $^{1}$ & Duplo leque & TJ60 11002 & 200 & 200 & 165 \\
\hline 4 & Tradicional $^{1}$ & Leque & XR11002 & 200 & 200 & 240 \\
\hline 5 & Modificada $30^{\circ}{ }^{2}$ & Duplo leque & TJ60 11002 & 200 & 400 & 165 \\
\hline 6 & Modificada $30^{\circ}{ }^{2}$ & Leque & XR11002 & 200 & 400 & 240 \\
\hline 7 & Modificada $45^{\circ} 2$ & Duplo leque & TJ60 11002 & 200 & 400 & 165 \\
\hline 8 & Modificada $45^{\circ} 2$ & Leque & XR11002 & 200 & 400 & 240 \\
\hline 9 & Parcela controle $^{* * *}$ & & & & & \\
\hline
\end{tabular}

${ }^{*}$ Testemunha sem a aplicação de fungicida durante todo o ciclo.

** Testemunha com a aplicação de fungicida antes do espigamento, para o controle de doenças foliares. ${ }^{* * *} \mathrm{Na}$ primeira época foram realizadas 7 aplicações de tebuconazole $\left(150\right.$ g.i.a.ha $\left.{ }^{-1}\right)$ durante a antese (30/09 a 11/10). Na segunda época foram realizadas 6 aplicações de tebuconazole (150 g.i.a.ha $\left.{ }^{-1}\right)$ durante a antese (06/10 a 19/10). A aplicação foi realizada utilizando pontas de pulverização tipo duplo leque TJ60 11002 com volume de calda de 200 L.ha ${ }^{-1}$

${ }^{1}$ Barra tradicional: bicos espaçados a cada $0,50 \mathrm{~m}$ posicionados na vertical.

${ }^{2}$ Barra modificada: corpos de 2 bicos espaçados a cada $0,50 \mathrm{~m} ; 30^{\circ}$ (um bico posicionado para frente e outro para trás com ângulo de $30^{\circ}$ em relação à vertical); $45^{\circ}$ (um bico posicionado para frente e outro para trás com ângulo de $45^{\circ}$ em relação à vertical).

${ }^{3}$ Diâmetro mediano volumétrico $(\mu \mathrm{m})$, estimado com informações do fabricante.

com o auxílio de um pulverizador portátil pressurizado com $\mathrm{CO}_{2}$. A velocidade de deslocamento foi de $1 \mathrm{~m} . \mathrm{s}^{-1}$ e a barra foi conduzida a $0,4 \mathrm{~m}$ acima das espigas. No dia da aplicação foi determinada a quantidade de anteras soltas e presas em 50 espigas coletadas ao acaso. Considerou-se como anteras soltas aquelas que permanecem expostas pendentes pelo filete e presas aquelas que permanecem aprisionadas na extremidade apical das glumas somente com o ápice visível.

Os tratamentos foram aplicados nos dias 06 e 10 de outubro, nas duas épocas, respectivamente. A colheita de grãos foi realizada nos dias 20 e 28 de novembro (primeira e segunda épocas, respectivamente).

$\mathrm{Na}$ primeira época de semeadura, a quantidade de anteras soltas/espiga foi de $45,9 \pm 18,8 \mathrm{e}$ de anteras presas/espiga de 5,3土3,5. Durante a aplicação,

a) Barra Tradicional

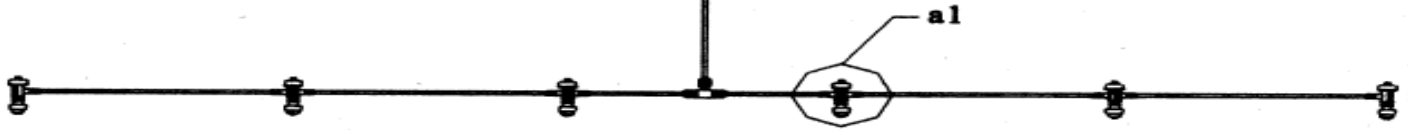

b) Barra Modificada
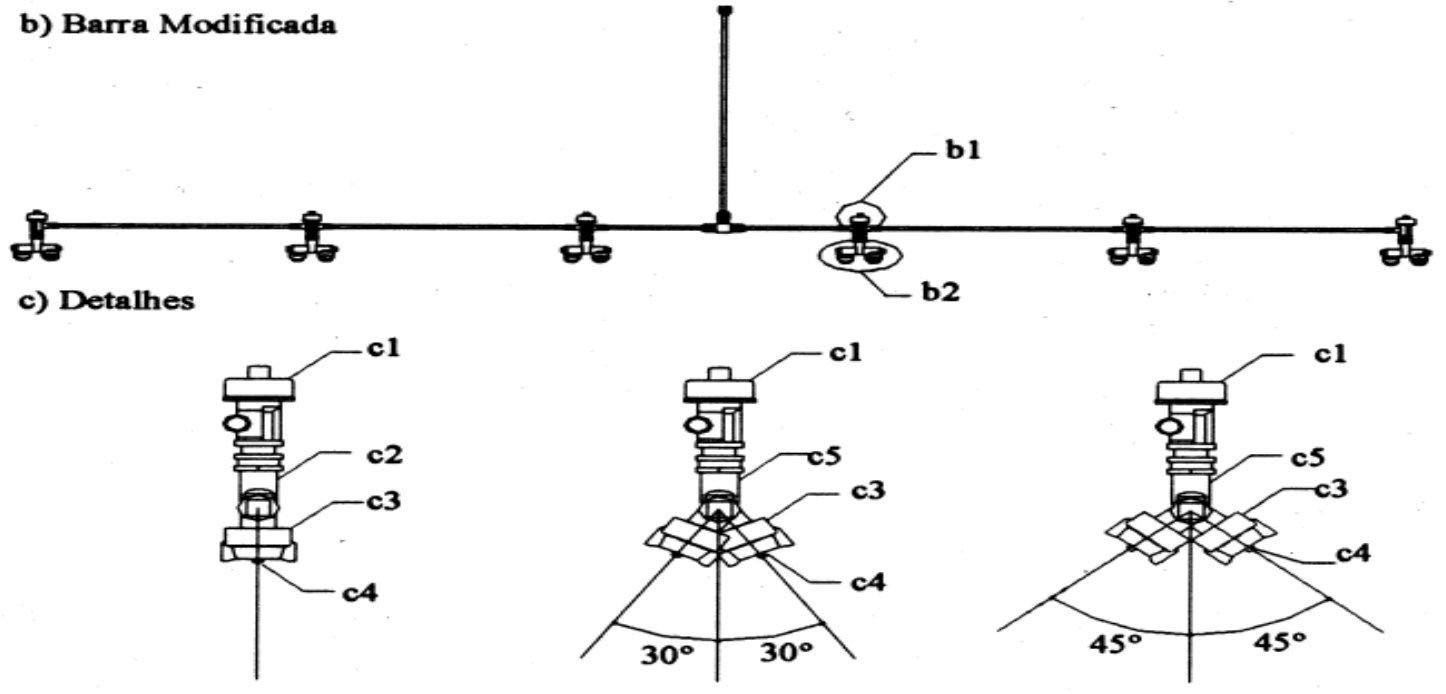

Figura 1 - Barras utilizadas e detalhe na posição dos bicos. a) Barra tradicional, a1) corpo de bico com válvula antigotejante; b) barra modificada, b1) válvula antigotejante, b2) corpo de bico giratório duplo (Código QJ8600-2-1/4 NYB - Spraying Systems); c) detalhes, c1) válvula antigotejante c2) corpo de bico c3) capa c4) ponta e c5) corpo de bico giratório. 
as condições climáticas foram: temperatura de $25^{\circ} \mathrm{C}$, umidade relativa do ar de $72 \%$ com rajadas de vento de até $10 \mathrm{~km} \cdot \mathrm{h}^{-1}$. Na segunda época de semeadura, a quantidade de anteras soltas/espiga foi de $36,6 \pm 14,8 \mathrm{e}$ de anteras presas/espiga $6,4 \pm 4,4$. Durante a aplicação, as condições climáticas foram: temperatura de $18{ }^{\circ} \mathrm{C}$, umidade relativa do ar $90 \%$, com rajadas de vento de até $4 \mathrm{~km} \cdot \mathrm{h}^{-1}$.

Nos dois experimentos, avaliaram-se a incidência, o número de espiguetas gibereladas, a severidade, o rendimento de grãos, o peso hectolítrico, o peso de mil sementes e a incidência de $\boldsymbol{F}$. graminearum em sementes. A incidência, o número de espiguetas gibereladas e a severidade, foram determinados no estádio 85 (ZADOCKS et al., 1974). A incidência foi determinada em $0,65 \mathrm{~m}^{2}$ considerando-se espiga doente a que apresentasse no mínimo duas espiguetas com sintoma. O número de espiguetas gibereladas foi determinado em 25 espigas coletadas ao acaso e convertido para porcentagem. A severidade foi calculada através da multiplicação da incidência pela porcentagem de espiguetas gibereladas dividindo-se por 100. Na primeira época, a colheita foi realizada em $5,1 \mathrm{~m}^{2} \mathrm{e}$ na segunda época em $6,12 \mathrm{~m}^{2}$. Para o cálculo do rendimento de grãos e do peso de mil sementes, a umidade dos grãos foi padronizada para $13 \%$. O peso de mil sementes foi determinado em 400 sementes por parcela. Determinou-se a incidência de $\boldsymbol{F}$. graminearum em 50 sementes por parcela. Após assepsia em hipoclorito de sódio, as sementes foram plaqueadas em meio seletivo a Fusarium (NASH \& SNYDER, 1962) e incubadas por sete dias em câmara de crescimento iluminada com luz fluorescente sob fotoperíodo de 12 horas com temperatura de $25 \pm 2{ }^{\circ} \mathrm{C}$.

O delineamento experimental foi o de blocos casualizados com quatro repetições. As médias foram submetidas à análise da variância e comparadas pelo teste de Duncan a 5\% de probabilidade de erro. Para a análise da variância da porcentagem de controle, os dados foram transformados para arco seno $\sqrt{\mathrm{x}}+0,5$.

\section{RESULTADOS E DISCUSSÃO}

Nos tratamentos 1 e 2, não foram observadas diferenças significativas entre as variáveis avaliadas, indicando que as doenças foliares não tiveram efeito sobre a intensidade da giberela, porém observa-se uma tendência de maior intensidade de giberela no tratamento 1, em que a intensidade de oídio foi maior do que no tratamento 2. A figura 2 mostra os resultados de controle da giberela nos diferentes tratamentos com aplicação de fungicida, em relação à testemunha sem aplicação (tratamento 1), considerando a incidência, o número de espiguetas gibereladas e a severidade da doença.

A incidência da doença foi de $73,3 \%$, a porcentagem de espiguetas gibereladas $52,9 \%$ e a severidade $38,8 \%$ para a primeira época. Na segunda época, a incidência foi de $70,2 \%$, a porcentagem de espiguetas gibereladas foi $49,4 \%$ e a severidade de $34,9 \%$. A aplicação do fungicida reduziu significativamente a quantidade de doença nas três variáveis mensuradas, nas duas épocas.

Com a utilização da parcela controle (tratamento 9), devido ao número de aplicações, esperava-se erradicar a doença. Porém, o melhor controle obtido foi de $91 \%$ com base na severidade. Embora na parcela controle, a doença não tenha sido erradicada, o incremento de rendimento foi de $53 \%$ e $64 \%$, nas épocas 1 e 2 , respectivamente. Isto sugere que a utilização do fungicida, ainda que não apresente níveis elevados de controle, demonstra apresentar potencial de aumentar o rendimento de grãos.

A percentagem de espiguetas gibereladas foi a variável que apresentou menor controle, podendo ser uma indicação de que o fungicida não impediu a colonização do fungo na espiga. Em relação aos tratamentos, observou-se controle superior somente nas parcelas controle. Os demais tratamentos, em todas as situações, comportaram-se de maneira semelhante.

Observou-se que o incremento médio do rendimento de grãos em relação à testemunha sem aplicação de fungicida foi de $26,7 \%$ e $30,3 \%$ para as épocas 1 e 2, respectivamente (Tabelas 2 e 3 ).

$\mathrm{O}$ pH dos grãos obtidos nas parcelas controle foi superior aos daqueles obtidos nos demais tratamentos. Os tratamentos também foram superiores às testemunhas, exceto na época 1 para o tratamento 3 . Nas duas épocas, o PMS foi superior na parcela controle e os outros tratamentos foram semelhantes às testemunhas (tratamentos 1 e 2). Na época 1, somente os tratamentos 6, 8 e 9 apresentaram incidência de $\boldsymbol{F}$. graminearum nas sementes menor que a testemunha (Tabela 2). Na época 2, apenas os tratamentos 8 e 9 apresentaram incidência do fungo nas sementes menor que a testemunha (Tabela 3). REIS (1988), estudando o curso da antese em cultivares de trigo observou que ela pode durar 4 dias em uma espiga, 12 dias em uma planta e 25 dias em uma lavoura. Recentemente, VARGAS et al. (2000) fizeram observações semelhantes. Por este motivo, a desuniformidade na antese, observada durante a aplicação, pode estar envolvida com o baixo controle alcançado com as aplicações dos fungicidas no campo (Figura 2). Concordando com as observações feitas por Mc MULLEN (1998), as aristas 


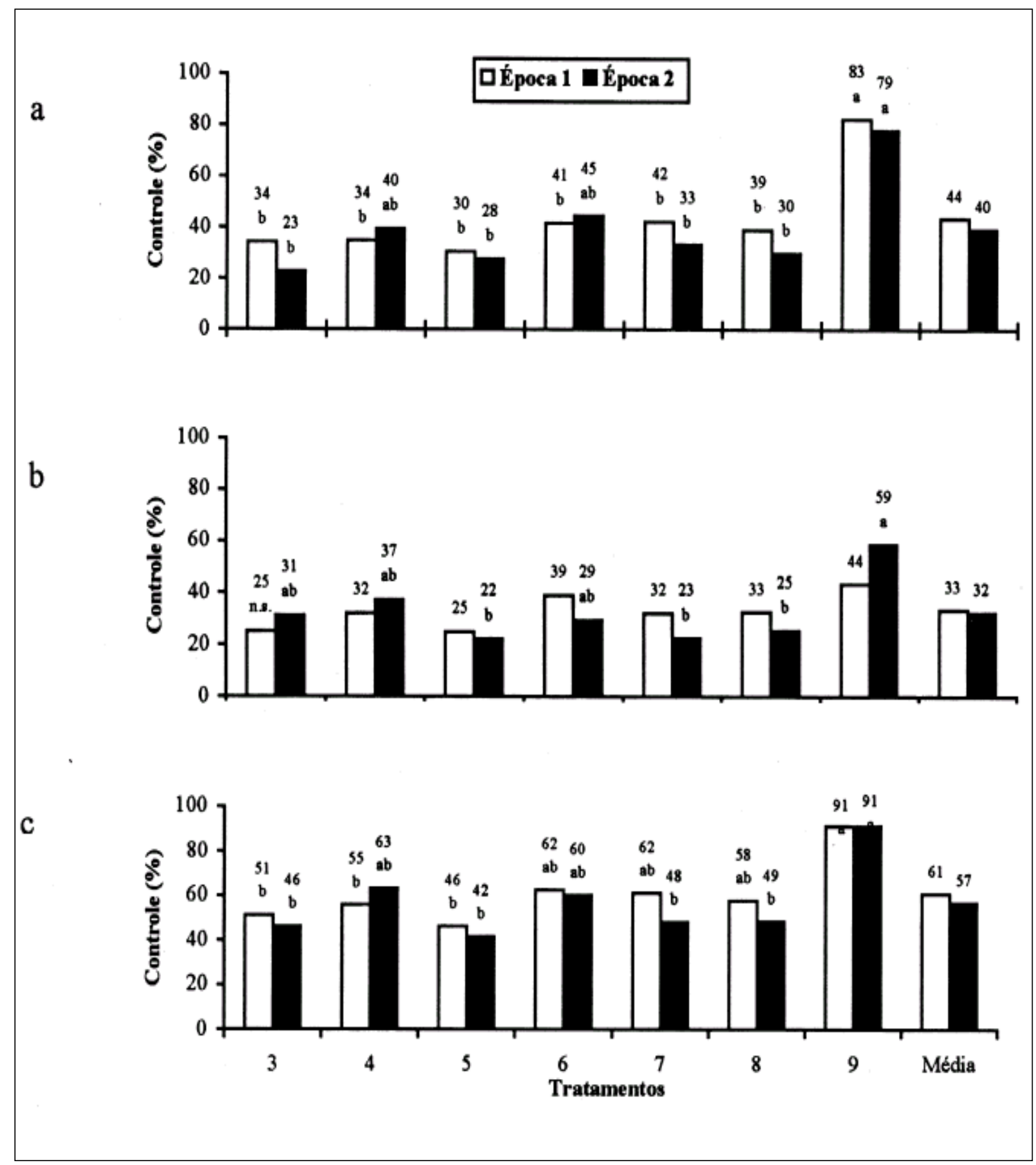

Figura 2 - Efeito de pontas de pulverização, posição destas na barra e volume de calda sobre o controle de giberela em trigo (Triticum aestivum). a) controle em relação a incidência; b) controle em relação ao número de espiguetas gibereladas; c) controle em relação severidade. Passo Fundo, RS, 2000. Colunas com a mesma letra, em cada época, não diferem significativamente pelo teste de Duncan (5\%), n.s. (não significativo).

podem dificultar a trajetória e deposição do fungicida nas anteras. Por outro lado, elas também podem dificultar a deposição dos esporos sobre as anteras, dificultando a infecção.
A baixa eficiência de controle, observada nos diferentes tratamentos (Figura 2), confirma os resultados obtidos por diversos pesquisadores (MARTIN\& JOHNSTON, 1982; MAULER-MACHNIK 
Tabela 2 - Efeito de pontas de pulverização, arranjo destas na barra e volume de calda sobre o rendimento de grãos $(\mathrm{RG})$, rendimento relativo $(\mathrm{RR})$, peso hectolítrico $(\mathrm{PH})$, peso de mil sementes (PMS) e incidência de Fusarium graminearum nas sementes (IS) em trigo (Triticum aestivum), semeado em 26 de junho, em Passo Fundo - RS, 2000 .

\begin{tabular}{|c|c|c|c|c|c|}
\hline $\begin{array}{c}\text { Tratamentos } \\
\text { (barra/ponta/volume de calda) }\end{array}$ & $\begin{array}{c}\mathrm{RG} \\
\left(\mathrm{kg} \cdot \mathrm{ha}^{-1}\right)\end{array}$ & $\begin{array}{l}\mathrm{RR} \\
(\%)\end{array}$ & $\begin{array}{c}\mathrm{PH} \\
(\mathrm{kg} / \mathrm{hL})\end{array}$ & $\begin{array}{c}\text { PMS } \\
(\mathrm{g})\end{array}$ & $\begin{array}{l}\text { IS } \\
(\%)\end{array}$ \\
\hline $1-$ Testemunha 1 & $2838 \mathrm{~d} *$ & - & $74,3 \mathrm{~d}$ & $30,6 \mathrm{bc}$ & $90,6 \mathrm{a}$ \\
\hline 2 - Testemunha 2 & $3149 \mathrm{c}$ & 11,0 & $74,6 \mathrm{~d}$ & $29,3 \mathrm{c}$ & 89,6 a \\
\hline 3 - Tradicional - duplo leque $/ 200$ & $3484 \mathrm{~b}$ & 22,8 & $75,6 \mathrm{~cd}$ & $31,0 \mathrm{bc}$ & $81,8 \mathrm{ab}$ \\
\hline 4 - Tradicional - leque $/ 200$ & $3625 \mathrm{~b}$ & 27,7 & $76,6 \mathrm{bc}$ & $31,8 \mathrm{~b}$ & $82,3 \mathrm{ab}$ \\
\hline 5 - Modificada $30^{\circ}-$ duplo leque $/ 400$ & $3578 \mathrm{~b}$ & 26,1 & $77,2 \mathrm{~b}$ & $31,3 \mathrm{bc}$ & $81,3 \mathrm{ab}$ \\
\hline 6 - Modificada $30^{\circ}-$ leque $/ 400$ & $3614 \mathrm{~b}$ & 27,3 & $76,6 \mathrm{bc}$ & $31,4 \mathrm{bc}$ & $77,1 \mathrm{~b}$ \\
\hline 7 - Modificada $45^{\circ}$ - duplo leque $/ 400$ & $3440 \mathrm{~b}$ & 21,2 & $76,4 \mathrm{bc}$ & $31,7 \mathrm{~b}$ & $81,3 \mathrm{ab}$ \\
\hline 8 - Modificada $45^{\circ}-$ leque $/ 400$ & $3573 \mathrm{~b}$ & 25,9 & $76,6 \mathrm{bc}$ & $32,2 \mathrm{~b}$ & $77,1 \mathrm{~b}$ \\
\hline 9 - Controle & $4312 \mathrm{a}$ & 51,9 & $79,8 \mathrm{a}$ & $35,6 \mathrm{a}$ & $64,1 \mathrm{c}$ \\
\hline Média & 3513 & 26,7 & 76,4 & 31,7 & 80,6 \\
\hline CV $(\%)$ & 4,92 & - & 1,06 & 4,19 & 8,43 \\
\hline
\end{tabular}

* Médias seguidas pelas mesmas letras nas colunas não diferem pelo teste de Duncan em nível de $5 \%$ de probabilidade de erro.
\& ZAHN, 1994; MESTERHÁZY \& BARTÓK, 1996; SUTY \& MAULER-MACHNIK, 1996; Mc MULLEN, 1998; SCHAAFSMA \& TAMBURIC-ILINCIC, 1998).

As diferentes pontas de pulverização e os seus arranjos na barra não proporcionaram melhor controle do que a barra tradicional com bico leque (11002) posicionado na vertical. Confirmam-se as observações feitas por Mc MULLEN (1998) de que a aplicação de fungicidas utilizando-se diferentes bicos hidráulicos pode proporcionar diferentes níveis de controle, mas geralmente, não são observadas diferenças significativas.

$\mathrm{Na}$ América do Norte, a aplicação para o controle de giberela é realizada com um bico leque (8002), embora não forneça boa cobertura das espigas.
Quando o mesmo bico foi utilizado com uma configuração diferente (dois bicos, um direcionado para frente e outro para trás), a cobertura foi melhor tanto nas espigas quanto em papel indicador (Mc MULLEN, 1998). Pelas observações durante a aplicação, a utilização dos bicos em configuração diferente (Tabela 1), aparentemente aumentou a cobertura das anteras, porém não foi verificado um aumento da eficiência do controle da doença.

Com a utilização de bicos voltados para frente e para trás, o volume de calda utilizado foi duplicado (de 200 L.ha-1 para $400{\mathrm{~L} . h a^{-1}}^{-}$. O aumento do volume deve ter melhorado a cobertura das espigas, porém a eficiência de controle não foi verificada, diferindo dos resultados obtidos por SAYLER (1999), que alcançou controle superior da giberela, quando utilizou volume de calda maior que 336 L.ha $^{-1}$.

Muitas vezes, a aplicação de fungicida específico para giberela não apresenta retorno satisfatório (REIS et al., 1996; Mc MULLEN et al. 1997). Embora com baixa intensidade de doença, a aplicação de fungicida proporcionou incremento de $21 \%$ no rendimento de grãos, confirmando os resultados obtidos por MARTIN \& JOHNSTON (1982) e WONG et al. (1992), que observaram que o rendimento de grãos é a variável mais afetada quando se aplica fungicida para o controle de giberela.

Na safra do ano 2000, o
custo do fungicida mais a aplicação

Tabela 3 - Efeito de pontas de pulverização, arranjo destas na barra e volume de calda sobre o rendimento de grãos (RG), rendimento relativo (RR), peso hectolítrico (PH), peso de mil sementes (PMS) e incidência de Fusarium graminearum nas sementes (IS) em trigo (Triticum aestivum), semeado em 08 de julho, em Passo Fundo - RS, 2000.

\begin{tabular}{|c|c|c|c|c|c|}
\hline $\begin{array}{l}\text { Tratamentos } \\
\text { (barra/ponta/volume de calda) }\end{array}$ & 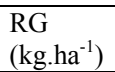 & $\begin{array}{l}\text { RR } \\
(\%)\end{array}$ & $\begin{array}{l}\mathrm{PH} \\
(\mathrm{kg} / \mathrm{hL})\end{array}$ & $\begin{array}{l}\text { PMS } \\
(\mathrm{g})\end{array}$ & $\begin{array}{l}\text { IS } \\
(\%)\end{array}$ \\
\hline $1-$ Testemunha 1 & $3085 \mathrm{c} *$ & - & $73,5 \mathrm{c}$ & $34,9 \mathrm{c}$ & $80,7 \mathrm{a}$ \\
\hline 2 - Testemunha 2 & $3280 \mathrm{c}$ & 6,3 & $73,9 \mathrm{c}$ & $35,3 \mathrm{bc}$ & $72,4 \mathrm{ab}$ \\
\hline 3 - Tradicional - duplo leque/200 & 3942 b & 27,8 & $75,0 \mathrm{~b}$ & $37,5 \mathrm{ab}$ & $64,1 \mathrm{bc}$ \\
\hline 4 - Tradicional - leque $/ 200$ & $3921 \mathrm{~b}$ & 27,1 & $75,0 \mathrm{~b}$ & $35,6 \mathrm{bc}$ & $70,3 \mathrm{ab}$ \\
\hline 5 - Modificada $30^{\circ}-$ duplo leque $/ 400$ & $3949 \mathrm{~b}$ & 28,0 & $74,8 \mathrm{~b}$ & $36,8 \mathrm{abc}$ & $71,4 \mathrm{ab}$ \\
\hline 6 - Modificada $30^{\circ}$ - leque $/ 400$ & $3957 \mathrm{~b}$ & 28,3 & $75,2 \mathrm{~b}$ & $37,4 \mathrm{ab}$ & $69,8 \mathrm{ab}$ \\
\hline 7 - Modificada $45^{\circ}$ - duplo leque $/ 400$ & $4047 \mathrm{~b}$ & 31,2 & $74,9 \mathrm{~b}$ & $37,0 \mathrm{abc}$ & $72,9 \mathrm{ab}$ \\
\hline 8 - Modificada $45^{\circ}$ - leque $/ 400$ & $3991 \mathrm{~b}$ & 29,4 & $75,2 \mathrm{~b}$ & $36,9 \mathrm{abc}$ & $58,3 \mathrm{c}$ \\
\hline 9 - Controle & $5055 \mathrm{a}$ & 63,9 & $77,9 \mathrm{a}$ & $38,6 \mathrm{a}$ & $37,0 \mathrm{~d}$ \\
\hline Média & 3914 & 30,3 & 75,0 & 36,7 & 66,3 \\
\hline CV $(\%)$ & 6,95 & - & 0,72 & 3,67 & 11,07 \\
\hline
\end{tabular}

* Médias seguidas pelas mesmas letras nas colunas, não diferem pelo teste de Duncan em nível de $5 \%$ de probabilidade de erro situou-se em torno de U\$ 24,00.ha- ${ }^{-1}$. Considerando-se o preço do trigo de U\$ 105,00/tonelada, o incremento de $229 \mathrm{~kg} \cdot \mathrm{ha}^{-1}$ equivale ao custo de uma aplicação de fungicida. Naquele ano (alta pressão de doença), o incremento médio do rendimento de grãos, nas parcelas tratadas, em relação a testemunha sem tratamento foi de $21 \%$ (em uma lavoura com potencial de rendimento de 3000 kg.ha-1 $\mathrm{o}$ incremento de grãos seria de $630 \mathrm{~kg} \cdot \mathrm{ha}^{-1}$ ). Desta forma, a aplicação de fungicida para o controle de giberela deve ser recomendada, já que o incremento no 
rendimento de grãos supera o custo da utilização do fungicida. Além disso, houve ainda, incremento do peso do hectolitro e redução da incidência do fungo nos grãos.

\section{CONCLUSÕES}

Os resultados obtidos, nas condições dos dois experimentos, permitem concluir que a substituição da ponta de pulverização de jato plano tradicional (leque 11002), por uma ponta de jatos em duplo leque (11002) não apresenta vantagens em relação ao controle da giberela, ao rendimento de grãos, ao peso do hectolitro e ao peso de mil sementes de trigo.

O aumento do volume de calda de 200 L.ha $^{-1}$ para 400 L.ha $^{-1}$ e a modificação da barra de pulverização com o direcionamento dos bicos $30^{\circ}$ ou $45^{\circ}$ para frente e para trás em relação à vertical não se justificaram pelos níveis de controle da doença, pelo rendimento de grãos e pelo peso hectolítrico obtidos.

Em lavouras com bom potencial de rendimento, justifica-se a aplicação de fungicida para o controle de giberela, uma vez que o incremento no rendimento de grãos supera o custo do tratamento.

Barras de pulverização tradicionais, com bicos espaçados em $0,50 \mathrm{~m}$, equipadas com pontas de jato plano (leque 11002), aplicando um volume de calda de 200 L.ha $^{-1}$ são adequadas para aplicação de fungicidas destinados ao controle da giberela em trigo.

\section{REFERÊNCIAS BIBLIOGRÁFICAS}

AGRIOS, G.N. Plant pathology. 3.ed. St. Paul : Academic, 1988. 803 p.

BAI, G.; SHANER, G. Scab of wheat: prospects for control. Plant Disease, St. Paul, v.78, p.760-766, 1994.

MARTIN, R.A.; JOHNSTON, H.W. Effects and control of fusarium diseases of cereal grains in the Atlantic Provinces. Canadian Journal of Plant Pathology, Ottawa, v.4, p.210216, 1982.

MAULER-MACHNIK, A.; ZAHN, K. Ear fusarioses in wheat - new findings on their epidemiology and control with Folicur (tebuconazole). Pflanzenschutz Nachrichten Bayer, Leverkusen, v.47, p.129-155, 1994.

Mc MULLEN, M.; JONES, R.; GALLENBERG, D. Scab of wheat and barley: a re-emerging disease of devastating impact. Plant Disease, St. Paul, v.81, p.1340-1348, 1997.

Mc MULLEN, M. Fungicide technology network of the national FHB initiative - 1998 Report. In: THE 1998 NATIONAL FUSARIUM HEAD BLIGHT FORUM, 1998, Michigan. Proceedings... Michigan : Michigan State University, 1998. p.47-50.

MESTERHÁZY, A.; BÁRTOK, T. Control of Fusarium head blight of weat by fungicides and its effect on the toxin contamination of the grains. Pflanzenschutz-Nachrichten Bayer, Leverkusen, v.49, p.181-198, 1996.

MILUS, E.A.; WEIGHT, C.T. Efficacy of Quadris and Benlate applications on wheat scab in Arkansas. In: THE 1998 NATIONAL FUSARIUM HEAD BLIGHT FORUM, 1998, Michigan. Proceedings... Michigan : Michigan State University, 1998. p.51-52

NASH, S.M.; SNYDER, W.C. Quantitative estimatives by plate counts of propagules of the bean root rot Fusarium in field soil. Phytopathology, St. Paul, v.52, p.567-572, 1962.

PARRY, D.W.; JENKINSON, P.; Mc LEOD, L. Fusarium ear blight (scab) in small grain cereals - a review. Plant Pathology, London, v.44, p.207-238, 1995.

REIS, E.M. Doenças do trigo III: giberela. 2.ed. São Paulo : E.M.REIS, 1988. 13p.

REIS, E.M.; BLUM, M.M.C.; CASA, R.T. Controle químico de Gibberella zeae em trigo, um problema de deposição de fungicidas em anteras. Summa Phytopathologic, São Paulo, v.22, p.3942, 1996.

REIS, E.M.; CASA, R.T.; FORCELINI, C.A. Doenças do trigo. In: KIMATI, H. et al. Manual de fitopatologia: doenças de plantas cultivadas. 3ed. São Paulo : Agronômica Ceres, 1997. p.725-736.

REIS, E.M.; FERNANDES, J.M.; PICININI, E.C. Estratégias para o controle de doenças do trigo. Passo Fundo : EmbrapaCnpt. 1988. 50p.

REUNIÃO DA COMISSÃO SUL-BRASILEIRA DE PESQUISA DE TRIGO, 2000. Cruz Alta, RS. Recomendações... Cruz Alta : Comissão Sul-Brasileira de Pesquisa de Trigo, 2000. $90 \mathrm{p}$.

SAYLER, T. NDSU research yields more clues for improving fungicide application techniques. 1997. Disponível em: <http://www.smallgrains.org.>. Acesso em: 14 jul. 1997.

SCHAAFSMA, A.W.; TAMBURIC-ILINCIC, L.; MILLER, J.D. The effect of agronomic practice on the accumalation of deoxynivalenol (DON) in winter wheat fields in Ontario, 1996-1997. In: THE 1998 NATIONAL FUSARIUM HEAD BLIGHT FORUM, 1998, Michigan. Proceedings... Michigan : Michigan State University. 1998. p.7-10.

SUTTON, J.C. Epidemiology of wheat head blight and maize ear rot caused by Fusarium graminearum. Canadian Journal of Plant Pathology, Ottawa, v.4, p.195-209, 1982.

SUTY, A.; MAULER-MACHNIK A. Fusarium head blight on wheat - new findings on the epidemiology and control of Gibberella zeae the teleomorph of Fusarium graminearum with Folicur. Pflanzenschutz-Nachrichten Bayer, Leverkusen, v.49, p.55-70, 1996. 
TUITE, J.; SHANER, G.; EVERSON R.J. Wheat scab in soft red winter wheat in Indiana in 1986 and its relation to some quality measurements. Plant Disease, St. Paul, v.74, p.959962, 1990.

VARGAS, P.R. et al. Simulação de epidemia de giberela em trigo. Fitopatologia Brasileira, Brasília, v.25, p.497-504, 2000.

WIESE, M. V. Compendium of wheat diseases. 2.ed. St.
Paul : The American Phytopathological Society, 1987. 106p.

WONG, L.S.L. et. al. Prevalence, distribution and importance of fusarium headblight in wheat in Manitoba. Canadian Journal of Plant Pathology, Ottawa, v.14, p.233-238, 1992.

ZADOCKS, J.C.; CHANG, T.T.; KONZAC, C.F. A decimal code for the growth stages of cereals. Weed Research, Oxford, v.14, p.415-421, 1974. 